(C) 2008 The Japan Society of Applied Physics

\title{
Generation of Second-Harmonic Bessel Beams by Transverse Phase-Matching in Annular Periodically Poled Structures
}

\author{
Solomon M. SAlTiEL ${ }^{1,2}$, Dragomir N. NeSheV ${ }^{1}$, Robert FisCHER ${ }^{1}$, \\ Wieslaw KrolikowsKi ${ }^{1}$, Ady ARIE ${ }^{3}$, and Yuri S. KIVSHAR ${ }^{1}$ \\ ${ }^{1}$ Nonlinear Physics Center and Laser Physics Center, Center for Ultra-high bandwidth Devices for Optical Systems (CUDOS), \\ Research School of Physical Sciences and Engineering, Australian National University, Canberra ACT 0200, Australia \\ ${ }^{2}$ Physics Department, Sofia University "St. Kl. Ohridski”, Sofia-1164, Bulgaria \\ ${ }^{3}$ School of Electrical Engineering, Faculty of Engineering, Tel-Aviv University, Ramat Aviv, Tel-Aviv, Israel \\ (Received January 26, 2008; accepted April 2, 2008; published online August 22, 2008)
}

We demonstrate experimentally the generation of second-harmonic conical waves via parametric frequency doubling in a twodimensional annular periodically poled nonlinear photonic structure fabricated in a stoichiometric lithium tantalate crystal. We apply the concept of nonlinear Bragg diffraction for explaining this effect, and show theoretically that in low-symmetry crystals this process results in generated second-harmonic waves being a superposition of the Bessel beams.

[DOI: 10.1143/JJAP.47.6777]

KEYWORDS: nonlinear optics, frequency conversion, harmonic generation, diffraction, scattering

\section{Introduction}

Nonlinear crystals with two-dimensional (2D) modulation of the sign of the second-order nonlinearity ${ }^{1,2}$ provide a natural extension of the well-known concept of one-dimensional (1D) periodically poled gratings used for achieving efficient phase matching (PM) in ferroelectric crystals (see, e.g., ref. 3 and references therein). Many kinds of such 2D nonlinear photonic structures with different pattern geometries have been proposed and experimentally tested including the five periodic 2D Bravais lattices: square, rectangular, hexagonal, centered rectangular, and oblique, ${ }^{4)}$ quasi-periodic, ${ }^{5)}$ or even random structures (see, e.g., refs. 6 and 7). In all these cases ${ }^{4-9)}$ the corresponding modulated structures have been excited in the direction perpendicular to the poling direction (in our notations, the $Z$-axis) and perpendicular to the walls of the anti-parallel domains. Exciting such artificially fabricated structures along the $Z$ axis (in the transverse direction with respect to the direction of a change of nonlinear properties of the media) leads to the generation of different kinds of the second-harmonic $(\mathrm{SH})$ scattered patterns. Here we study, both theoretically and experimentally, the transverse excitation of annularly periodically poled structures introduced previously by Kasimov et al. ${ }^{9)} \mathrm{We}$ show that the $\mathrm{SH}$ output radiation appears in the form of a superposition of conical waves that represent the far field of the Bessel beams propagating inside the crystal. The reason for the appearance of such multi-ring structures is explained by using the concept of nonlinear Bragg diffraction. Some of the results presented here have been discussed previously in refs. 10 and 11 .

The effect of Bragg diffraction is usually observed in the propagation of waves in a medium with a periodic change of the optical refractive index. ${ }^{12)}$ For certain angles of incidence, the partially reflected waves add coherently and form strong beams propagating outside the sample at the angles $\beta_{m}=\sin ^{-1}(m \lambda / \Lambda)$ where $\lambda$ is the wavelength, $\Lambda$ is the modulation period, and $m$ is an integer. Importantly, similar Bragg diffraction can also occur in media with a spatially homogeneous optical refractive index but periodically varying nonlinear properties. This effect, known as nonlinear Bragg diffraction, was first discussed by Freund, ${ }^{13}$ ) and it was observed experimentally in naturally laminated crystals exhibiting non-regularity and dispersion of nonlinear domains. ${ }^{14,15)}$ Recently, nonlinear diffraction has also been reported in the second- and third-harmonic generation in the presence of a transient optically-induced 1D grating. ${ }^{16)}$ Nonlinear diffraction is observed also in photonic crystals with simultaneous modulation of both linear and nonlinear optical properties. ${ }^{17,18)}$ Nonlinear Bragg diffraction with multi-order generation of $\mathrm{SH}$ is observed in one dimensional gratings formed by a series of waveguides made by proton exchange. ${ }^{19)}$

In this paper we report on theoretical and experimental studies of conical second-harmonic generation (SHG) from a 2D nonlinear photonic structure fabricated in a periodically poled stoichiometric lithium tantalate (SLT) crystal with annular periodic modulation of the second-order nonlinearity. We observe that the transverse illumination of a radially periodic structure by a strong Gaussian beam exactly in the center of the structure leads to multiple conical emission of $\mathrm{SH}$ waves outside the sample.

The idea of the experiment is shown in Fig. 1. The observed $\mathrm{SH}$ waves form a set of rings in the far-field zone, and the angles of their propagation satisfy the well-known Bragg relation, i.e., they are determined by a ratio of the $\mathrm{SH}$ wavelength to the modulation period of the nonlinearity. We study the polarization properties of the diffracted $\mathrm{SH}$ rings and demonstrate that they are determined, in a complex way, by the input polarization and the second-order nonlinear susceptibility tensor. In a general case, the polarization varies with the azimuthal angle of the ring. Moreover, we show theoretically that in such an annular quasiphase-matched (QPM) structure the SH light field inside the sample is composed of Bessel beams of different widths and orders, so that the diffraction rings shown in Fig. 1 are the far-field images of the $\mathrm{SH}$ Bessel beams generated inside the nonlinear crystal.

\section{Phase Matching Conditions}

We consider a nonlinear photonic structure with a $2 \mathrm{D}$ periodic modulation of the second-order nonlinear response but otherwise homogeneous linear refractive index. As discussed in the Introduction, 2D QPM nonlinear photonic 


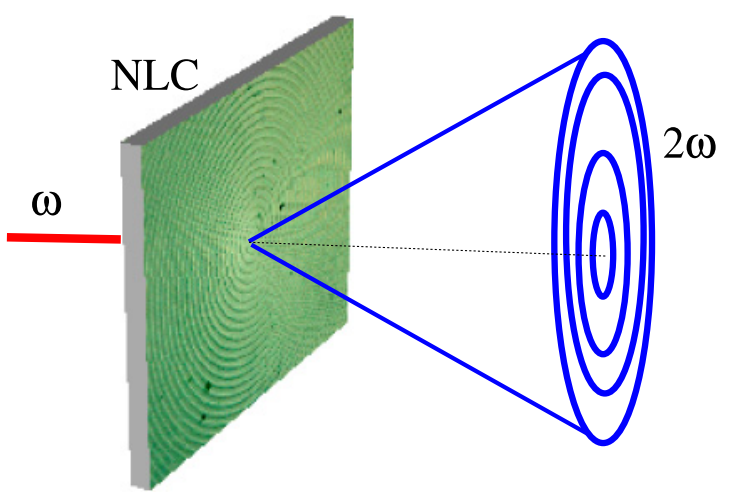

Fig. 1. (Color online) Schematic of the experiment (NLC identifies the nonlinear crystal). The annular periodically poled structure shown is a photo of the etched surface of the sample before polishing.

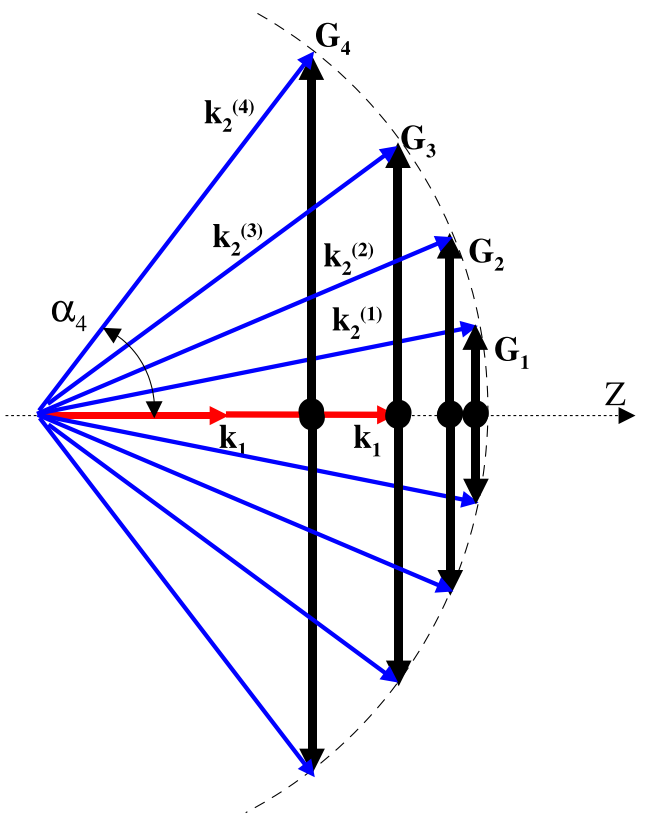

Fig. 2. (Color online) Phase matching diagram of the SHG parametric processes in the transverse periodically poled gratings. $\mathbf{G}_{m}$ are the reciprocal vectors of the structure.

structures have been suggested and explored exclusively for the longitudinal geometry when the input beam is directed perpendicularly to the domains with periodically varying second-order nonlinearity. In this study we explored a novel type of the so-called transverse PM geometry for the 2D nonlinear photonic crystal with a circularly periodic poled structure, as shown in Fig. 1. To explain the SH diffraction rings observed in the experiment we consider the PM conditions shown in Fig. 2 for the corresponding SHG process. The sample with an annular QPM structure ${ }^{9)}$ with the period $\Lambda=7.5 \mu \mathrm{m}$ is excited with an intense laser beam. The beam is directed along the symmetry axis of the structure which coincides with the $Z$ (optical) axis of the crystal. The fundamental wave can propagate in this direction only as an ordinary wave. However, the generated SH waves can be both ordinarily and extraordinarily polarized. This means that, in general, there will be two sets of the $\mathrm{SH}$ wave vectors $\mathbf{k}_{2 \mathrm{o}}$ and $\mathbf{k}_{2 \mathrm{e}}$ for the geometry shown in Fig. 2. The general vectorial PM conditions are:

$$
\begin{aligned}
& 2 \mathbf{k}_{1 \mathrm{o}}+\mathbf{G}_{m}+\Delta \mathbf{k}_{m}=\mathbf{k}_{2 \mathrm{e}}^{(m)}\left(\alpha_{m}\right), \\
& 2 \mathbf{k}_{1 \mathrm{o}}+\mathbf{G}_{m}+\Delta \mathbf{k}_{m}=\mathbf{k}_{2 \mathrm{o}}^{(m)},
\end{aligned}
$$

where $\mathbf{k}_{1}, \mathbf{k}_{2 \mathrm{o}}^{(m)}$, and $\mathbf{k}_{2 \mathrm{e}}^{(m)}\left(\alpha_{m}\right)$ are the wave-vectors of the fundamental and $\mathrm{SH}$ waves, $\mathbf{G}_{m}$ is the $m$-order of the reciprocal vector of the $\chi^{(2)}$ modulation $\left|G_{m}\right|=m(2 \pi / \Lambda)$, $\Delta \mathbf{k}_{\mathrm{m}}$ is the phase mismatch, and $m$ is the order of the generated $\mathrm{SH}$ wave. It is convenient to split these PM conditions $^{20,21)}$ into two, namely the transverse condition,

$$
\begin{aligned}
2 n_{2 \mathrm{o}}(\lambda) \sin \alpha_{m, \mathrm{o}} & =m \lambda / \Lambda, \\
2 n_{2 \mathrm{e}}\left(\lambda, \alpha_{m, \mathrm{e}}\right) \sin \alpha_{m, \mathrm{e}} & =m \lambda / \Lambda,
\end{aligned}
$$

and the longitudinal condition:

$$
\begin{aligned}
n_{2 \mathrm{o}}(\lambda) \cos \alpha_{m, \mathrm{o}}-n_{1 \mathrm{o}}(\lambda) & =\Delta n_{m, \mathrm{o}}, \\
n_{2 \mathrm{e}}\left(\lambda, \alpha_{m}\right) \cos \alpha_{m, \mathrm{e}}-n_{1 \mathrm{o}}(\lambda) & =\Delta n_{m, \mathrm{e}}
\end{aligned}
$$

With a correct choice of the fundamental wavelength or crystal temperature, a given diffraction order can be made to be longitudinally phase-matched with $\Delta n_{m, \mathrm{o}}=0$ or $\Delta n_{m, \mathrm{e}}=0$. As an example, in the situation depicted in Fig. 2, the third-order diffraction $\mathrm{SH}$ process is phasematched (i.e., $\Delta k_{3}=0$ and $\Delta n_{3}=0$ ), and for this interaction both PM conditions are satisfied simultaneously. Inside the crystal, the propagation angles for the ordinary transversely phase-matched waves are found from relation (3)

$$
\alpha_{m, \mathrm{o}}=\sin ^{-1}\left(m \lambda_{2} / n_{2, \mathrm{o}} \Lambda\right),
$$

where $\lambda_{2}$ is the SH wavelength. It is interesting to point out that the internal PM angle does not depend on the index of refraction of the fundamental wave. Moreover, the outside angles $\beta_{m, \mathrm{o}}$ (for $Z$-cut sample) do not dependent on any of the indices of refraction of the sample:

$$
\beta_{m, \mathrm{o}}=\sin ^{-1}\left(m \lambda_{2} / \Lambda\right) .
$$

Equation (8) is exactly the Bragg condition for the $\mathrm{SH}$ beam diffracted by the linear structure with the period $\Lambda$. To find the angles corresponding to the PM for the extraordinary SH waves, we must solve eq. (4) numerically. However, for small birefringence, as is the case of SLT, we have

$$
n_{2 \mathrm{e}}\left(\lambda, \alpha_{m, \mathrm{e}}\right)=n_{2 \mathrm{o}}(\lambda)\left[1-\delta \sin ^{2}\left(\alpha_{m, \mathrm{e}}\right)\right],
$$

where $\delta(\lambda)=\Delta N(\lambda) / n_{20}(\lambda)$ is the normalized difference between the main values of the ordinary and extraordinary index of refraction in this crystal. Note $\delta<0.01$ for the SLT crystal. ${ }^{22)}$ With eq. (9) the PM conditions (4) yields

$$
\sin \alpha_{m, \mathrm{e}}=\sin \alpha_{m, \mathrm{o}}\left[1+\delta(\lambda) \sin ^{2}\left(\alpha_{m, \mathrm{e}}\right)\right] .
$$

From eq. (10) we find for the external angles $\beta_{m, o}$ :

$$
\sin \beta_{m, \mathrm{e}} \cong \sin \beta_{m, \mathrm{o}}\left[1-\delta^{2} \sin ^{4}\left(\alpha_{m, \mathrm{o}}\right)\right] .
$$

Since birefringence of SLT crystals is quite small, eq. (11) shows that the difference between the two angles $\beta_{m, \mathrm{o}}$ and $\beta_{m, \mathrm{e}}$ for the propagation of extraordinary and ordinary $\mathrm{SH}$ waves is very small as well $\left(<\delta^{2}\right)$. For the 0.5 -mm-long sample it is not possible to resolve experimentally the spatial difference between the ordinary and extraordinary rings. However, from the polarization measurements it is easy to see that each ring is, in fact, a mixture of two rings: ordinary and extraordinary. 
The discussion so far considered the PM conditions in a single propagation plane. Since the structure has an azimuthal symmetry, the properties of the $\mathrm{SH}$ are the same for any azimuthal angle $\varphi$. Consequently, the $\mathrm{SH}$ radiation appears in the form of concentric cones with cone angles $\beta_{m}$, as shown in Fig. 1.

\section{Effective Nonlinearities and Polarization Properties}

The emission rings possess interesting polarization properties defined by the tensor of the second-order nonlinearity of the lithium tantalate crystal. This crystal belongs to the $3 m$ symmetry point group. For the fundamental beam propagating along the axis $Z$, the tensor of second order nonlinearity has the following relevant nonzero components: $d_{z x x}=d_{z y y}$, and $d_{x x y}=d_{y x x}=-d_{y y y}$. In periodically poled crystals belonging to the $3 m$ symmetry group $d_{x x y}, d_{y x x}, d_{y y y}$ also change sign. ${ }^{23,24)}$ As we pointed out earlier, two types of the SH processes are possible: Type I [(oo-e), two ordinary waves generate an extraordinary $\mathrm{SH}$ wave] and Type 0 [(oo-o), two ordinary waves generate an ordinary $\mathrm{SH}$ wave)]. Effective nonlinearities can be found by calculating first the polarization of the nonlinear media $\mathbf{P}(2 \omega)=\mathbf{d}^{(2)} \mathbf{E E}$. Let us define the input direction of polarization of wave $\mathbf{A}$ propagating along $Z$ as follows: $\mathbf{A}=[\cos \gamma, \sin \gamma, 0]$. Then the three components of the nonlinear polarization of the media are

$$
\begin{aligned}
& P_{x} / A^{2}=2 d_{x x y} \sin \gamma \cos \gamma=-2 d_{y y y} \sin 2 \gamma, \\
& P_{y} / A^{2}=\left(d_{y y y} \sin ^{2} \gamma+d_{y x x} \cos ^{2} \gamma\right)=-d_{y y y} \cos 2 \gamma, \\
& P_{z} / A^{2}=\left(d_{z y y} \cos ^{2} \gamma+d_{z x x} \sin ^{2} \gamma\right)=d_{z y y} .
\end{aligned}
$$

The unit vectors of generated ordinary and extraordinary $\mathrm{SH}$ waves are given as

$$
\begin{aligned}
& \mathbf{o}=[\sin \varphi,-\cos \varphi, 0], \\
& \mathbf{e}=[-\cos \varphi \cos \alpha,-\sin \varphi \cos \alpha, \sin \alpha] .
\end{aligned}
$$

After projecting the polarization of the nonlinear media on these unit vectors, we find the following expressions for the effective nonlinearities responsible for the two processes [oo-o] and [oo-e]:

$$
\begin{aligned}
& d_{\mathrm{eff}}^{(\mathrm{o})}=d_{y y y} \cos (\varphi+2 \gamma), \\
& d_{\mathrm{eff}}^{(\mathrm{e})}=d_{y y y} \sin (\varphi+2 \gamma) \cos \alpha+d_{z y y} \sin \alpha,
\end{aligned}
$$

where $\varphi$ is the azimuthal angle, $\alpha$ is the diffraction angle inside the crystal, and $\gamma$ is the angle that defines the orientation of the input beam polarization plane (for the fundamental wave polarized along $X, \gamma=0$ ). The definitions of angles $\varphi$ and $\alpha$ are given in Fig. 3 .

As illustrated in Fig. 3, the polarization vector of the ordinary $\mathrm{SH}$ wave is always perpendicular to the plane formed by any of the $k_{2}$ vectors and $Z$-axis. Then the cone formed by the ordinary wave will be azimuthally polarized. In contrast, the polarization vector of the extraordinary $\mathrm{SH}$ wave is always in the plane formed by any of the $k_{2}$ vectors and the $Z$-axis. Then the cone formed by the extraordinary wave is radially polarized. At the output of the crystal for small angles $\alpha$, for each azimuthal angle $\varphi$ the two cones overlap quite well and they propagate outside the crystal as a single cone with polarization that depends on the input polarization.

The azimuthal intensity distribution of the ordinary and extraordinary SH depends on the input polarization defined

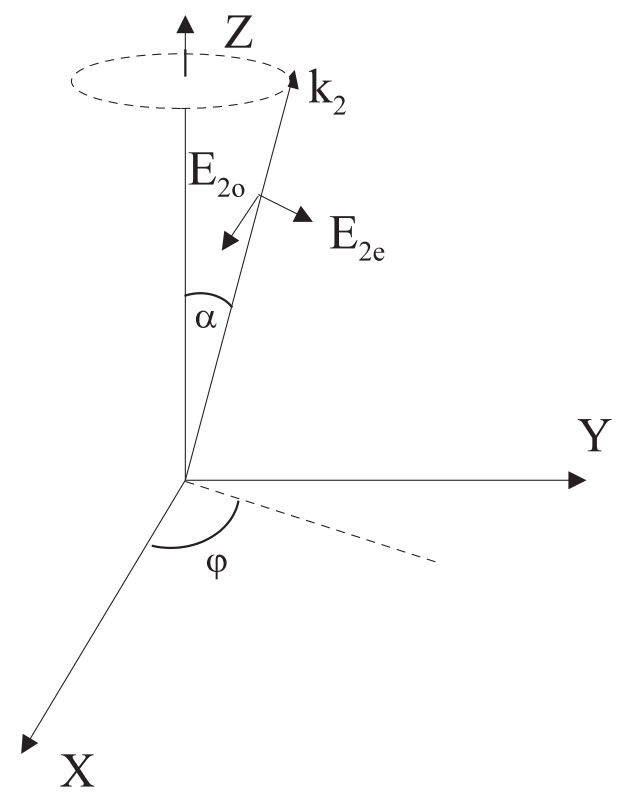

Fig. 3. Definitions of the angles and vectors for the derivation of the effective nonlinearities.

by the angle $\gamma$. Since the two rings are orthogonally polarized the total intensity is given as

$$
I_{2 \omega, m} \propto\left\{S^{(\mathrm{e})}\left[d_{\mathrm{eff}}^{(\mathrm{e})}\right]^{2}+S^{(\mathrm{o})}\left[d_{\mathrm{eff}}^{(\mathrm{o})}\right]^{2}\right\} I_{1}^{2} g_{m}^{2} L^{2},
$$

where $\quad S^{(\mathrm{o}, \mathrm{e})}=\sin ^{2}\left(\pi L / 2 L_{\mathrm{coh}}^{(\mathrm{o}, \mathrm{e})}\right) /\left(\pi L / 2 L_{\mathrm{coh}}^{(\mathrm{o}, \mathrm{e})}\right)^{2}, \quad L_{\mathrm{coh}}^{(\mathrm{o}, \mathrm{e})}=\pi /$ $\Delta k_{m}^{(\mathrm{o}, \mathrm{e})}$, and $g_{m}=2 /(\pi m) \sin (\pi m D)$, where $D$ is the duty factor.

For small angles $\alpha$, when we can neglect the difference in the refractive indices of the two waves, the polarization of the whole ring is linear. For $\gamma=0$ and $\pi / 2$, polarization for any $\varphi$ coincides with $Y$ - and, for $\gamma=\pi / 4$ with $X$-directions. The intensity of the total ring does not depend on the orientation of the input polarization. For relatively large angles $\alpha$, each of the rings propagates with its own phase velocity $\left[n_{2 \mathrm{o}}>n_{2 \mathrm{e}}(\alpha)\right]$ and at slightly different internal angles. In this case, the output polarization as a function of the azimuthal angle also depends on the actual overlap of the two rings at the output surface since the ordinary and extraordinary rings are tangentially and radially polarized, respectively. For large values of $\alpha$ and for relatively long crystals, the second term of eq. (18) cannot be neglected, and $m$-th diffraction order should appear as a doublet of cross-polarized rings.

The wave vectors of $\mathrm{SH}$ waves emitted by the annular periodically poled SLT lie on a cone with a conical angle $\alpha$. It is known that a superposition of an infinite number of plane waves with the wave vectors lying on a cone results in the formation of a Bessel beam. ${ }^{25,26)}$ A more detailed analysis of the SHG process in a structure with azimuthal symmetry shows that, the SH wave inside the crystal has the form of a single Bessel beam or superposition of the several Bessel beams. In the case of strontium barium niobate (SBN) crystals discussed earlier, ${ }^{20}$ the symmetry of this crystal ( $4 \mathrm{~mm}$ ) caused the $\mathrm{SH}$ wave inside the crystal to be represented by just a first-order Bessel beam with a perfect radial polarization. We performed similar analysis for the case of $\mathrm{LiTaO}_{3}$ or $\mathrm{LiNbO}_{3}$, ( $3 m$ point group symmetry). The 
beam structure for these crystals is more complicated due to the contribution of several components of the $\chi^{(2)}$ tensor. However, it still can be represented in terms of a superposition of the low-order Bessel functions with the angularly modulated intensity. Following the formalism of ref. 20 we arrive at the following result for $x$ and $y$ components of amplitude $\mathbf{V}^{(\mathrm{o})}$ of an ordinary SH wave:

$$
\begin{aligned}
V_{x}^{(\mathrm{o})}(\mathbf{r})= & 2 \pi A^{2} d_{y y y}\left[-J_{0}\left(\xi_{0}\right) \sin \varphi \cos \beta\right. \\
& \left.+\frac{J_{1}\left(\xi_{0}\right)}{\xi_{0}} \sin (\varphi+\beta)\right] \exp \left(i k_{\mathrm{o}} z\right), \\
V_{y}^{(\mathrm{o})}(\mathbf{r})= & 2 \pi A^{2} d_{y y y}\left[J_{0}\left(\xi_{0}\right) \cos \varphi \cos \beta\right. \\
& \left.-\frac{J_{1}\left(\xi_{0}\right)}{\xi_{0}} \cos (\varphi+\beta)\right] \exp \left(i k_{\mathrm{o}} z\right) .
\end{aligned}
$$

By analogy, the $x$ and $y$ components of the extraordinary $\mathrm{SH}$ wave amplitude are

$$
\begin{aligned}
V_{x}^{(\mathrm{e})}(\mathbf{r})= & 2 \pi A^{2}\left\{\left[d_{1} J_{0}\left(\xi_{\mathrm{e}}\right) \sin \beta+i d_{2} J_{1}\left(\xi_{\mathrm{e}}\right)\right] \cos \varphi\right. \\
& \left.-d_{1} \frac{J_{1}\left(\xi_{\mathrm{e}}\right)}{\xi_{\mathrm{e}}} \sin (\varphi+\beta)\right\} \exp \left(i k_{\mathrm{e}} z\right), \\
V_{y}^{(\mathrm{e})}(\mathbf{r})= & 2 \pi A^{2}\left\{\left[d_{1} J_{0}\left(\xi_{\mathrm{e}}\right) \sin \beta+i d_{2} J_{1}\left(\xi_{\mathrm{e}}\right)\right] \sin \varphi\right. \\
& \left.+d_{1} \frac{J_{1}\left(\xi_{\mathrm{e}}\right)}{\xi_{\mathrm{e}}} \cos (\varphi+\beta)\right\} \exp \left(i k_{\mathrm{e}} z\right) .
\end{aligned}
$$

In these equations $\rho=\left(x^{2}+y^{2}\right)^{1 / 2}$ denotes the transverse radial coordinate, $\xi_{\mathrm{o}, \mathrm{e}}=k_{2 \mathrm{o}, \mathrm{e}} \rho \sin \alpha, \quad d_{1}=d_{y y y} \cos \alpha$, $d_{2}=d_{z y y} \sin \alpha$, and $\beta=\varphi+2 \gamma$. At the output surface of the sample $(z=L)$, the two components of the total field are

$$
\begin{aligned}
V_{x, y}(\rho, \varphi, L)= & V_{x, y}^{(\mathrm{o})}(\rho, \varphi, z) \exp \left(i k_{\mathrm{o}} L\right) \\
& +V_{x, y}^{(\mathrm{e})}(\rho, \varphi, z) \exp \left(i k_{\mathrm{e}} L\right)
\end{aligned}
$$

These formulas can be simplified in the case of small emission angles when $\tan \alpha_{m} \ll 1$ :

$$
\mathbf{V}^{(\mathrm{e})}(\rho, \varphi, z)=2 \pi A^{2} d_{y y y} J_{\mathrm{o}}(\xi)\left(\boldsymbol{u}_{x} \sin 2 \gamma+\boldsymbol{u}_{y} \cos 2 \gamma\right),
$$

when $\xi \approx \xi_{\mathrm{e}} \approx \xi_{\mathrm{o}}$. It is clear that the beam represented by eq. (25) is linearly polarized, and its intensity is independent of the azimuthal coordinate

$$
I_{2 \omega}=4 d_{y y y}^{2} \pi^{2} A^{4} J_{0}^{2}(\xi) .
$$

As we show in the next section, this predicted azimuthal dependence agrees very well with experimental observations.

\section{Experimental Results and Discussion}

The stoichiometric lithium tantalate (SLT) sample is described in ref. 9. It is $0.49 \mathrm{~mm}$ thick, with a QPM period of $7.5 \mu \mathrm{m}$. The duty factor varies inside the sample from 0.7 at the $(Z+)$ surface to 0.8 at the $(Z-)$ surface. The $(Z+)$ and $(Z-)$ surfaces are polished. Small $(20-\mathrm{nm})$ deep grooves remain after the polishing. They cause slight diffraction of the fundamental beam at an angle twice less than the angle of the first order SH diffraction. The corresponding diffraction efficiency is less than $3 \%$. To observe the effect of the generation of conical SH waves, we employed an experimental setup that consists of a Mira Ti:sapphire laser with a regenerative amplifier. It delivers $140 \mathrm{fs}$ pulses with

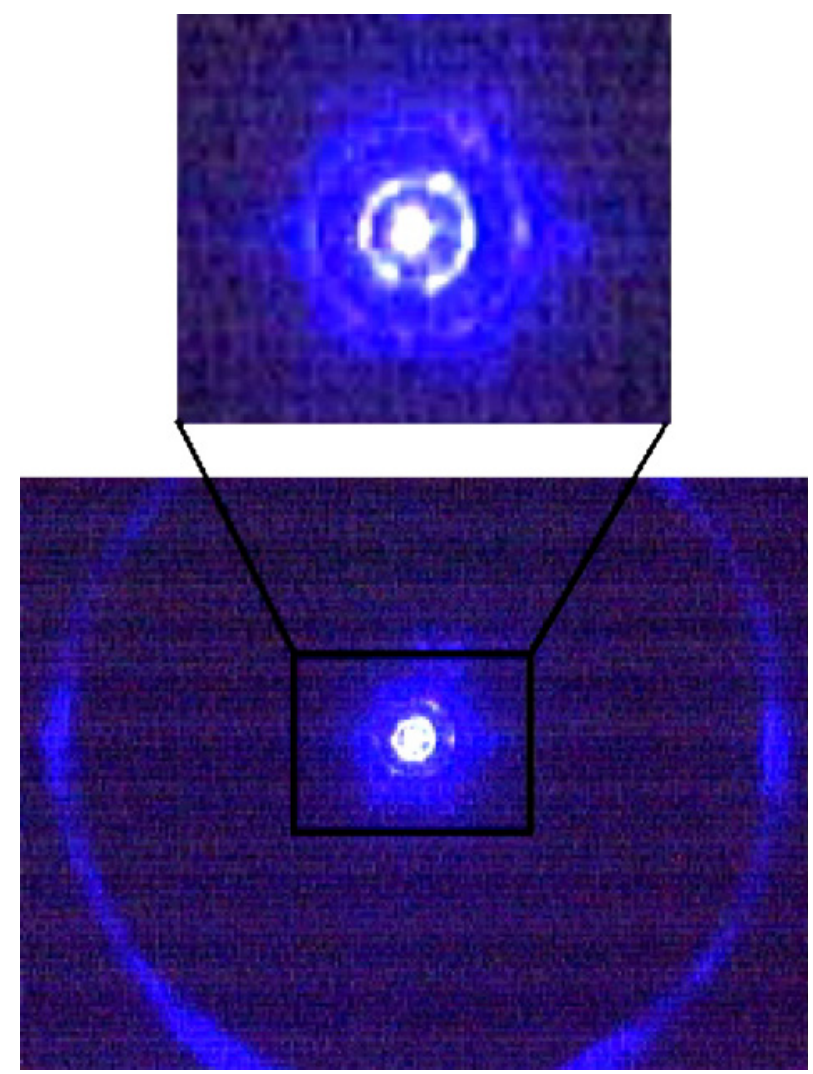

Fig. 4. (Color online) Observed $\mathrm{SH}$ rings with laser tuned at $822 \mathrm{~nm}$. 1st order, 2nd order, and 13th order (phase-matched) rings are seen. The fundamental beam is exactly in the center of the structure.

repetition rate of $250 \mathrm{kHz}$. The time-bandwidth product is 0.49 . Average output power is $740 \mathrm{~mW}$. The radiation was focused in the sample with a $30 \mathrm{~cm}$ lens exactly in the center of the structure. The waist size of the beam at the position of the sample measured with the knife-edge method is $147 \mu \mathrm{m}$ (FWHM). The measured beam diameter corresponds to about $100 \mathrm{GW} / \mathrm{cm}^{2}$ of intensity. The observed SH rings with a laser tuned at $822 \mathrm{~nm}$ are shown in Fig. 4. The cone angle is measured by recording the ring diameter as a function of the distance from the sample. In this way we determine that the largest ring is phase matched for the 13th order. Its cone angle is $45.6^{\circ}$ as measured (and it is slightly different from the predicted angle of $45.4^{\circ}$ ) by simply re-scaling the lowerorder diffraction patterns. In the same calculation (using the data for the refractive index of $\mathrm{SLT}^{22)}$ ) of the PM for the 13th order SH wave gives a PM wavelength $836 \mathrm{~nm}$ with a cone angle of $46.4^{\circ}$. We attribute this difference in the wavelength and angle to the absence of good Sellmeier coefficients for the ordinary index of refraction of SLT.

The PM curve is shown in Fig. 5 together with the theoretical prediction based on the model from ref. 20 for a duty factor of 0.748 . We observe strong similarities in the shape of the experimental curve and the theoretical prediction. The difference in the PM wavelength $(822.2 \mathrm{~nm}$ for experimental, and $836 \mathrm{~nm}$ for theoretical), as we already discussed, is most likely due to uncertainty in the value of the ordinary refractive index caused by the lack of accurate corresponding Sellmeier coefficients. In Fig. 5(a) we verify a quadratic dependence of the $\mathrm{SH}$ power of the phase matched 13th order $\mathrm{SH}$ ring as a function of the input power. 

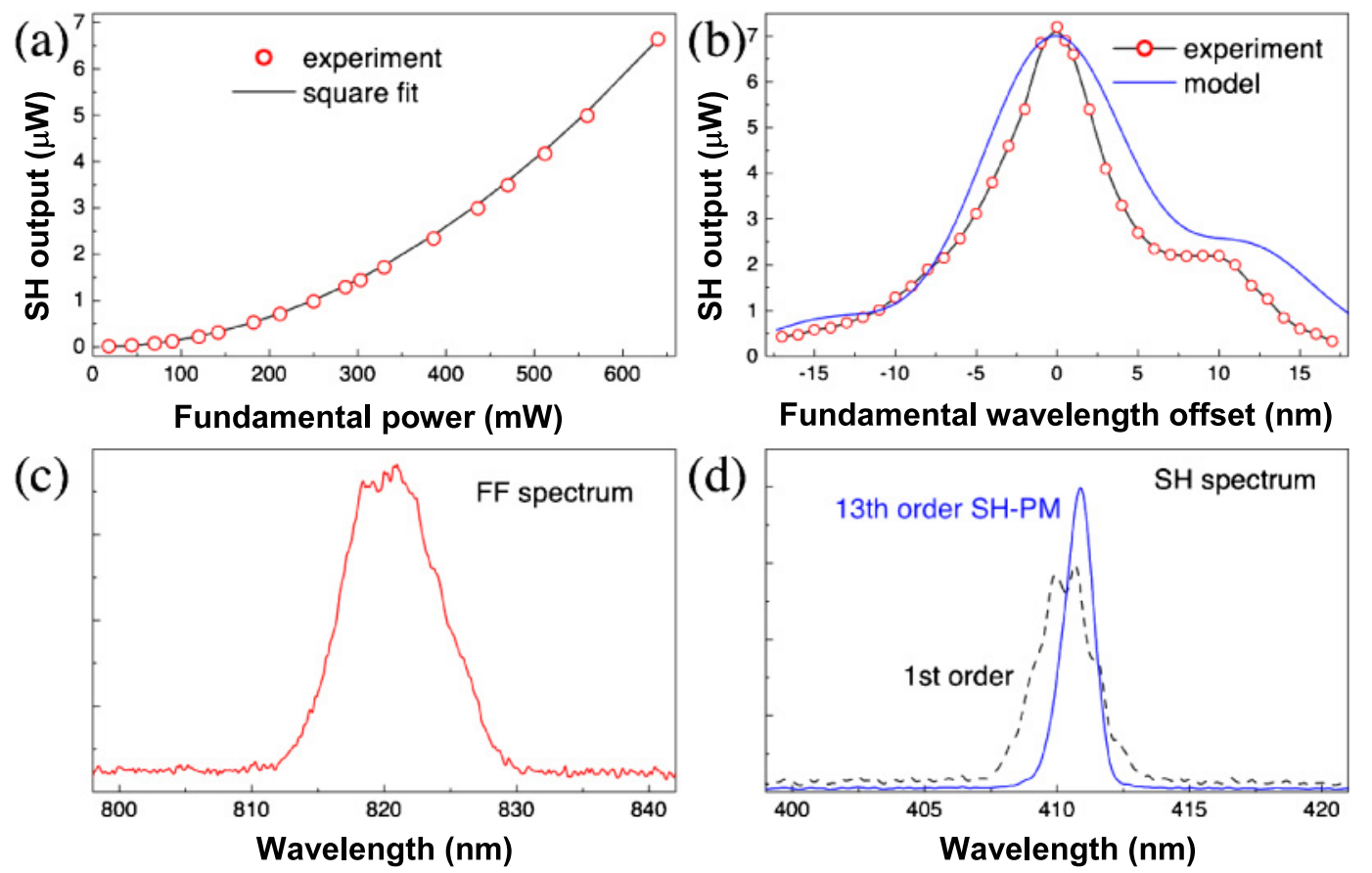

Fig. 5. (Color online) (a) Quadratic dependence of the 13th order SH ring. (b) Experimental PM curve and theoretical prediction normalized to the maximum of the experiment. (c) Spectrum of the fundamental radiation. (d) Spectra of the SH rings for nonphasematched (1st order) and phase-matched (13th order) SH rings.

From these data obtained at $640 \mathrm{~mW}$ fundamental power after taking into account all losses, we determine the internal efficiency for the phase-matched 13th order SH ring, $\eta_{13}=0.0036 \%$. Such low efficiency is attributed to the fact that we use 13th order QPM interaction and the duty factor varies inside the crystal leading to an effective length 2-3 times less than the real sample thickness. The measured efficiency of the $\mathrm{SH}$ power generated in the first-order ring is 220 times less than the 13th. Our estimation shows that for a 1-mm-long sample operating in the regime of the first order QPM with a homogeneous $50 \%$ duty factor, $10 \%$ efficiency of $\mathrm{SH}$ generation could be achieved.

The spectra are shown in Figs. 5(c) and 5(d). The spectral width of the fundamental is $8 \mathrm{~nm}$. The FWHM of the spectra of the SH ring in the case of PM of the 13th order is $1.3 \mathrm{~nm}$; i.e., almost 2 times less than expected for a stationary process. This means that the SHG process takes place in group-velocity mismatch conditions. Indeed, the calculated group-velocity-mismatch length is $0.092 \mathrm{~mm}$ for a $140 \mathrm{fs}$ fundamental pulse width, which is half of the expected effective length of the sample, and the expected reduction of the spectrum (lengthening of the pulse) is also correspondingly 0.5 ( 2 times). In contrast to the phase-matched 13th order ring, the spectra widths of non-phase matched (first and second order) rings are not reduced and are close to the prediction for the stationary process, $\Delta \omega_{r m, \mathrm{SH}}=\sqrt{2} \Delta \omega_{r m, \mathrm{FF}}$. The reason for this is that the length of interaction is short and equal to the coherence length, which is of the order of a few micrometers.

The rings are sensitive to an axial alignment of the fundamental beam. With off-center pumping, the brightness of SH diffracted spots is higher and number of the observed diffracted orders increases [see Fig. 6 (top)]. For a horizontal (vertical) shift of the sample, the rings transform into horizontally (vertically) aligned spots. This occurs because the structure resembles a $1 \mathrm{D}$ periodically poled grating formed from the sectors situated close to the horizontal (vertical) diameter of the rings. The two strong points at both sides of the top photo in Fig. 6 correspond to the 13th order nonlinear SH diffraction. The modifications of the diffraction pattern as a function of the horizontal shift for only the phase-matched 13th order ring are shown in Figs. 6(a)-6(c). In fact, consideration of the PM conditions shown in Fig. 2 remains the same for off-center pumping with the difference that $\mathrm{SH}$ scattering takes place in one plane only.

In Figs. 6(d)-6(e) we show the experimental intensity distribution of the 1st and 13th order $\mathrm{SH}$ rings. The theoretical prediction for the 13th order $\mathrm{SH}$ ring is given by eq. (19), and it is depicted in Fig. 6(f). We observe a good agreement between the theoretical prediction and experimental results which confirms the correctness of the model.

Another important parameter is the polarization state of the generated SH rings. Some experimental results are shown in Fig. 7. As discussed in the theoretical section, the model predicts that each ring consists in fact of two rings, "ordinary" and "extraordinary". Since these two constituent rings are spatially unresolved and overlap perfectly outside the sample, they form a single composite ring with a polarization state that depends on the phase difference between the ordinary and extraordinary waves inside the sample. For lower order rings the predicted polarization of the composite ring is always linear. This is illustrated in Fig. 7(a) where it is seen that the first-order $\mathrm{SH}$ ring is linearly polarized when the input fundamental wave is linearly polarized. This agrees very well with the eqs. (17) and (18) as well as eqs. (20)-(23) in the limit of $\alpha \rightarrow 0$. When the input beam is polarized along either the $X$ or $Y$ axis, the SH conical wave is polarized along $Y$-axis. If the input fundamental beam is polarized at $45^{\circ}$ with respect to 

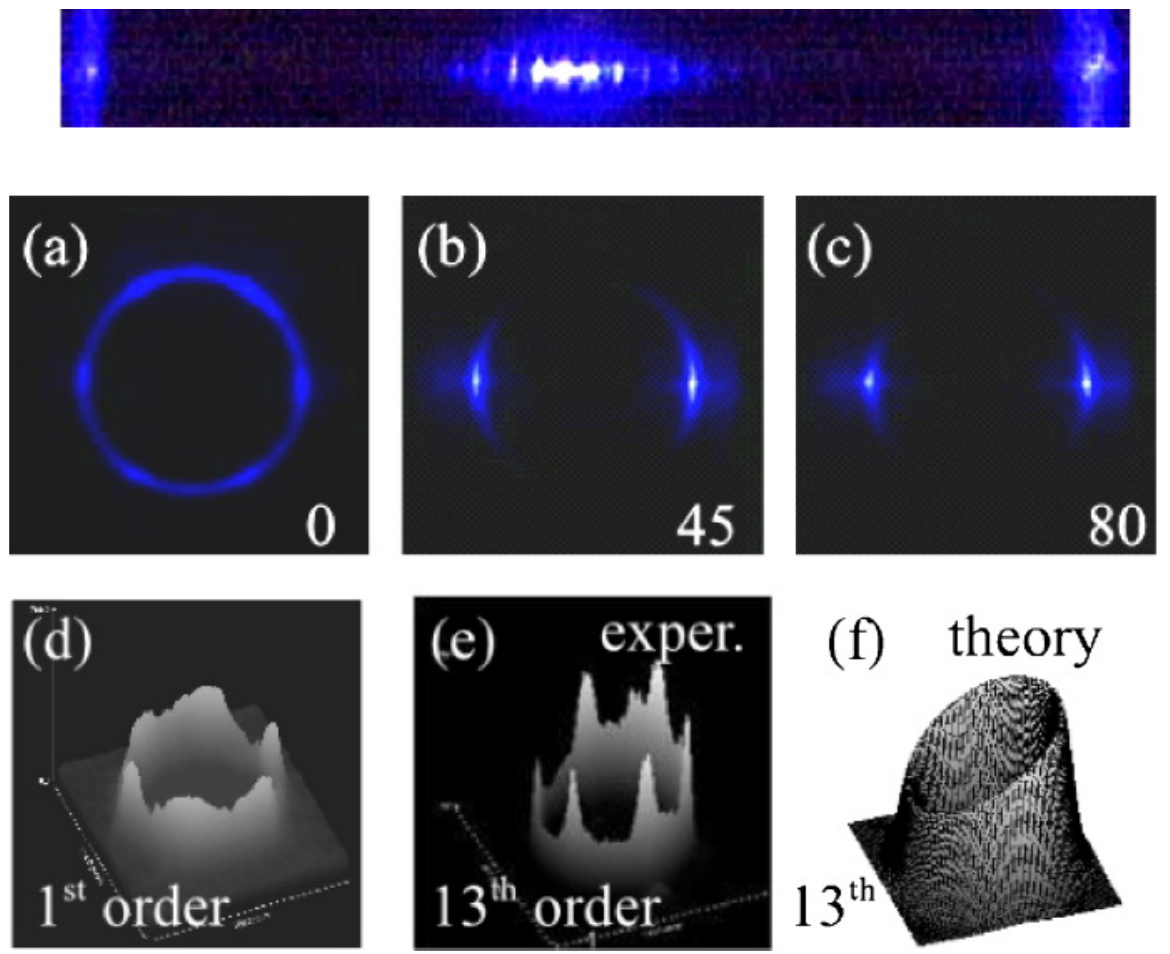

Fig. 6. (Color online) Top: Diffraction patterns with off-center excitation: shown are first 7 orders and 13th order SH nonlinear diffraction spots. (a-c) Modification of the diffraction pattern as a function of the horizontal shift for the phase matched SH ring ( $m=13$ ). Numbers indicate the shift in $\mu \mathrm{m}$. The $X$-axis of the samle is horizontal. (d) Experimental intensity distribution of $m=1$ SH ring. (e) Experimental intensity distribution of $m=13 \mathrm{SH}$ ring. (f) Theoretical prediction for the azimuthal intensity distribution of the $m=13 \mathrm{SH}$ ring based on eq. (19).

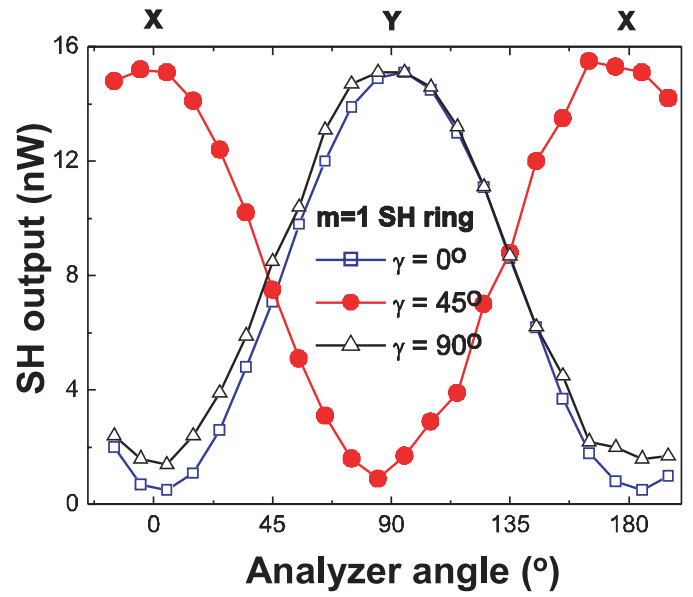

(a)

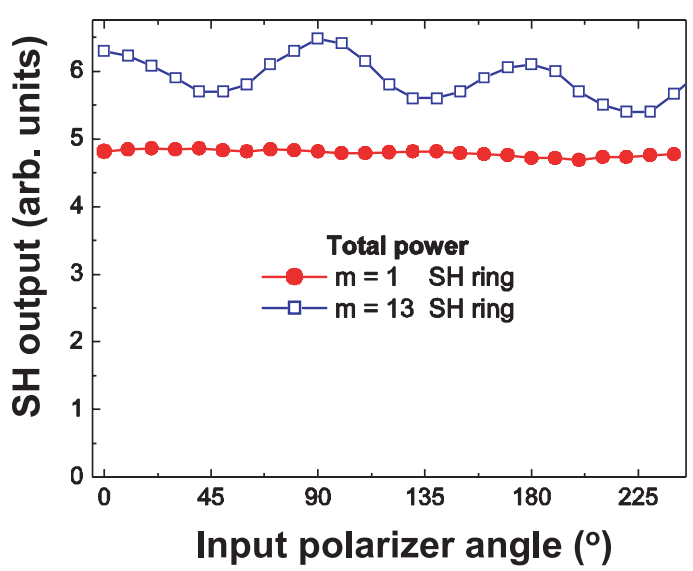

(b)

Fig. 7. (Color online) Polarization properties of the SH rings: (a) Power of the 1st order SH ring as a function of the analyzer angle; (b) Total power of the 1st and 13th order SH rings as a function of the input polarization angle.

these axes, the output SH conical wave is polarized along $X$. For arbitrary orientation $\gamma$ of the input polarization, we observe that the polarization of the first-order ring is oriented at the an angle $2 \gamma+\pi / 2$.

For the total power of the lower-order rings, the model (19) predicts insensitivity to the input polarization. The experimental straight line shown in Fig. 7(b) perfectly confirms this. For the higher-order $\mathrm{SH}$ rings the polarization state becomes more complex and the output power of the $\mathrm{SH}$ depends on the input polarization, as evidenced by oscillating curve in Fig. 7(b).
As can be seen from the photos [e.g., Figs. 6(a), 6(d), and $6(\mathrm{e})]$, the $\mathrm{SH}$ rings exhibit an additional azimuthal modulation with six well-defined peaks. These peaks are insensitive to input polarization and intensity and can be observed even in the regime of linear diffraction of the fundamental beam on the surface relief induced by the poling pattern. Therefore, we believe that those peaks are most likely induced by the discrete character of the domains boundaries resulting from the well-known ability of the ferroelectric domains in the $\mathrm{SLT}^{9,27)}$ to retain their hexagonal shape. 


\section{Conclusions}

We have studied theoretically and experimentally the generation of conical $\mathrm{SH}$ waves from 2D annular nonlinear photonic structures. We have shown that the observed phenomena can be explained by employing the concept of nonlinear Bragg diffraction combined with the conditions for both longitudinal and transverse phase matching. We have demonstrated that each of the generated waves represents a superposition of the Bessel beams. We have also studied the polarization properties of the generated conical $\mathrm{SH}$ radiation and demonstrated how they depend on crystal symmetry and cone angle. The geometry discussed here may enable the generation of the conical harmonics in other systems including acoustic waves in dielectrics and matter waves in optical lattices.

\section{Acknowledgments}

The work has been supported by the Australian Research Council and the Israeli Science Foundation, grant no. 960/05. We are gratefull for the assistance of D. Kasimov, A. Bruner, P. Shaier, and D. Eger in producing the SLT sample. Solomon Saltiel thanks the Nonlinear Physics Center for a warm hospitality and support.

1) V. Berger: Phys. Rev. Lett. 81 (1998) 4136.

2) N. G. R. Broderick, G. W. Ross, H. L. Offerhaus, D. J. Richardson, and D. C. Hanna: Phys. Rev. Lett. 84 (2000) 4345.

3) M. M. Fejer, G. A. Magel, D. H. Jundt, and R. L. Byer: IEEE J. Quantum Electron. 28 (1992) 2631.

4) A. Arie, N. Habshoosh, and A. Bahabad: Opt. Quantum Electron. 39 (2007) 361.

5) R. Lifshitz, A. Arie, and A. Bahabad: Phys. Rev. Lett. 95 (2005) 133901.
6) J. J. Romero, D. Jaque, J. Garca Sole, and A. A. Kaminskii: Appl. Phys. Lett. 78 (2001) 1961.

7) J. J. Romero, C. Arago, J. A. Gonzalo, D. Jaque, and J. Garcia Sole: J. Appl. Phys. 93 (2003) 3111

8) J. R. Kurz, A. M. Schober, D. S. Hum, A. J. Saltzman, and M. M. Fejer: IEEE J. Sel. Top. Quantum Electron. 8 (2002) 660.

9) D. Kasimov, A. Arie, E. Winebrand, G. Rosenman, A. Bruner, P. Shaier, and D. Eger: Opt. Express 14 (2006) 9371.

10) S. M. Saltiel, D. N. Neshev, W. Krolikowski, R. Fischer, A. Arie, and Yu. S. Kivshar: Phys. Rev. Lett. 100 (2008) 103902.

11) S. M. Saltiel, D. N. Neshev, W. Krolikowski, R. Fischer, A. Arie, and Yu. S. Kivshar: Proc. SPIE 6801 (2007) 680113

12) S. A. Akhmanov and S. Yu. Nikitin: Physical Optics (Clarendon Press, Oxford, U.K., 1997) Chap. 16

13) I. Freund: Phys. Rev. Lett. 21 (1968) 1404.

14) G. Dolino: Phys. Rev. B 6 (1972) 4025.

15) Y. Le Grand, D. Rouede, C. Odin, R. Aubry, and S. Mattauch: Opt. Commun. 200 (2001) 249.

16) T. Schneider and J. Reif: Phys. Rev. A 65 (2002) 023801.

17) C. Comaschi, G. Vecchi, A. M. Malvezzi, M. Patrini, G. Guizzetti, M. Liscidini, L. C. Andreani, D. Peyrade, and Y. Chen: Appl. Phys. B 81 (2005) 305.

18) A. M. Malvezzi, F. Cattaneo, G. Vecchi, M. Falasconi, G. Guizzetti, L. C. Andreani, F. Romanato, L. Businaro, E. D. Fabrizio, A. Passaseo, and M. D. Vittorio: J. Opt. Soc. Am. B 19 (2002) 2122.

19) T. Suhara, H. Tazaki, and H. Nishihara: Electon. Lett. 25 (1989) 1326.

20) S. Saltiel, W. Krolikowski, D. Neshev, and Yu. S. Kivshar: Opt. Express 15 (2007) 4132.

21) S. P. Tewari, H. Huang, and R. W. Boyd: Phys. Rev. A 54 (1996) 2314.

22) M. Nakamura, S. Higuchi, S. Takekawa, K. Terabe, Y. Furukawa, and K. Kitamura: Jpn. J. Appl. Phys. 41 (2002) L465.

23) A. Ganany, A. Arie, and S. M. Saltiel: Appl. Phys. B 85 (2006) 97.

24) G. A. Magel, M. M. Fejer, and R. L. Byer: Appl. Phys. Lett. 56 (1990) 108.

25) J. Durnin: J. Opt. Soc. Am. A 4 (1987) 651

26) Z. Bouchal and M. Olivik: J. Mod. Opt. 42 (1995) 1555.

27) V. Shur, E. Shishkin, I. Baturin, A. Chernykh, D. Kuznetsov, A. Lobov, A. Shur, M. Dolbilov, and K. Gallo: http://eprints.soton.ac.uk/ $42411 /$ 